\title{
Effects of psychological intervention on glucose metabolism in patients with type 2 diabetes mellitus accompanying depression in community
}

\author{
Jun Xue, Danhua Shi, Mei Ao, Lei Li, Zhaoxia Feng, Guomei Lv, Xiaojing Cao \\ Community Health Service Center of Friendship Office, The Third Affiliated Hospital of Inner Mongolia Medical University, \\ Baotou, Inner Mongolia, China
}

Received: September 12, 2017

Accepted: October 29, 2017

Online Published: December 10, 2017

DOI: $10.14725 /$ dcc.v4n4p6

URL: http://dx.doi.org/10.14725/dcc.v4n4p6

\begin{abstract}
Objective: To explore the effect of psychological intervention on glucose metabolism in patients with type 2 diabetes mellitus (T2DM) accompanying depression in community.

Methods: Four hundred and ten cases of T2DM patients in the Community Health Service Center of Friendship Office (Baotou) were chosen as the research objects. The evaluation and analysis were made according to Zung self-rating depression scale (SDS), and 176 cases of T2DM patients with depression were found out in the community. 176 cases of T2DM patients with depression were divided into the experimental group and the control group by use of random number table, with 88 cases in each group. The control group was treated with conventional therapy, while the experimental group was given psychological intervention treatment on the basis of conventional therapy. After 3-month psychological intervention, fasting blood glucose (FPG), two-hour postprandial blood glucose (PG2h), glycosylated hemoglobin (HbA1c) and Zung self-rating Depression Scale (SDS) were measured in two groups. In two months after psychological intervention stopped, the follow-up was conducted to determine the levels of FPG, PG2h and HbA1c, and to assess the level of depression and quality of life. Statistical analysis was performed by use of SPSS 17.0 statistical software.

Results: After 3-month psychological intervention, the levels of FPG, PG2h and HbA1c in the experimental group were significantly lower than those in the control group, the depression index was significantly lower than that in the control group as well, the difference was statistically significant $(p<.01)$. In two months after psychological intervention stopped, glucose metabolism indicators in the experimental group were significantly lower than those in the control group; the depression index was dramatically lower than that in the control group, the difference was of statistical significance $(p<.01)$.

Conclusions: Psychological intervention can effectively improve the depression symptoms and glucose metabolism indicators in T2DM patients with depression in community, but it needs a long-term persistence.
\end{abstract}

Key Words: Type 2 diabetes mellitus, Depression, Psychological intervention

Social psychological factors affect the development and prognosis of type 2 diabetes. Negative social psychological factors threaten patients' physical and psychological health, and result in a series of psychological problems such as de- pression, anxiety, cognitive disorders, etc. It is remarkably obvious that cognitive function is declined in patients with diabetes, which is related to the progress of diabetes and glucose control. Decline in cognitive function makes it uneasy

*Correspondence: Jun Xue; E-mail: 46753717@qq.com; Address: Community Health Service Center of Friendship Office, The Third Affiliated Hospital of Inner Mongolia Medical University, Baotou, Inner Mongolia, China. 
for patients to reach the glucose control standard, and increases medication dosage and varieties; patients may take medicine repeatedly if they forget taking them already, so that the risk of hypoglycemia will be increased eventually. Studies confirm that the risk of depression in T2DM patients is twice higher than that in non-T2DM patients, and independent of age, gender and other diseases. Depression can in turn lead to poor glucose control and decreased compliance. As elderly people are more likely to encounter the above-mentioned problems, it is recommended to perform a functional assessment while diagnosing diabetes, in order to control blood glucose and treat related diseases much better. ${ }^{[1]}$ Therefore, it is crucial to early detect depressive symptoms in patients with diabetes and provide them with a reasonable psychological intervention. This topic is intended to study the effect of psychological intervention on T2DM patients with depression in community.

\section{Data and methods}

\subsection{Data source}

Friendship Community in Baotou had a population of 10,050 , with 822 people suffering from diabetes mellitus. 410 cases of T2DM patients were chosen in Friendship Community, including 220 males and 190 females; the youngest was 30 years old and the oldest was 86 years old, the average age was $(67.10 \pm 10.21)$ years. Diagnostic criteria and classification of type 2 diabetes mellitus conformed to China Guideline for Type 2 Diabetes (2013). ${ }^{[2]}$ All patients volunteered in this experiment, excluding patients with mental illness, cognitive disorders and acute complications.

\subsection{Grouping and methods}

\subsubsection{Depression rating}

In this study, depression rating was conducted with Zung self-rating depression scale (SDS), with good reliability and validity. First of all, the staff received special training on psychological examination. Then, trained and qualified staff gave out questionnaires uniformly in a standardized and independent way. Lastly, each questionnaire was checked on a case-by-case basis to ensure that the data were qualified and valid. This scale, mainly reflecting 4 groups of specific symptoms, including psycho-emotional symptoms, somatic dyskinesia, psycho-motor disorders and psychological disorders etc., easy to operate, can intuitively reflect subjective feelings in nearly one week. There are 20 items totally in the SDS questionnaire, 10 items for thesis and 10 items for antithesis. The thesis item is rated in ascending order from 1 to 4 , while the antithesis one is rated in descending order from 4 to 1 . The sum of all scores from each item is taken as the total score. Each item is rated from 1 to 4 , and the highest score is 80 points. Depression index refers to the value resulted from the total score divided by 80 : value less than 0.5 indicates no depression; value ranging from 0.50 to 0.59 indicates mild depression; value ranging from 0.60 to 0.69 stands for moderate depression; value more than 0.70 suggests severe depression. In the first part of this research, 410 patients with type 2 diabetes mellitus were evaluated and analyzed by use of self-rating depression scale; it was proved that 176 cases of T2DM patients suffered from depression. In the second part of the research, 176 cases of T2DM patients with depression who were enrolled in the first part, were divided into the control group and the experimental group by use of random number table, with 88 cases in each group. The differences in age and gender of 2 groups were not statistically significant.

\subsubsection{Measurement methods}

Two groups of patients were both given conventional therapy of anti-diabetic drugs. Meanwhile, the experimental group was also given psychological treatment, but the control group wasn't. Anxiety degree, level of depression, quality of life, fasting blood glucose (FPG), two-hour postprandial blood glucose (PG2h) and glycosylated hemoglobin (HbA1c) were measured before treatment, after 3-month psychological intervention and in 2 months after psychological intervention stopped.

\subsubsection{Data collection}

First of all, investigators should explain the purpose of the investigation to respondents and make sure they volunteered in this research. And the investigators patiently explained to the respondents how to fill in the questionnaire. For poorlyeducated and illiterate patients, the investigators should interpret and explain each item in detail, so that the investigators would fill in the questionnaire by deputy after the respondents responded accordingly. Questionnaires were taken back after completing the form. It was required to check and verify these forms carefully, to ensure that the information was true and qualified.

\subsubsection{Indicator observation}

Before and after treatment, someone will score Zung selfrating depression scale and detect fasting blood glucose, two-hour postprandial blood glucose (both by use of German Bayer Brite micro-glucose meter) and glycosylated hemoglobin. 


\subsection{Statistical methods}

SPSS 17.0 statistical software was applied to the data treatment, the measurement data were represented by $\bar{x} \pm \mathrm{s}$. The comparison of age was made by use of $t$-test, and the comparison of gender was made by use of chi-square $\left(\chi^{2}\right)$ test. For glucose metabolism indicators and depression index, ANOVA of repeated measurement and LSD- $t$ test were applied, and the differences $(p<.05)$ were statistically significant.

\section{Results}

The mean age of the experimental group was (56.57 \pm $12.35)$, while the average age of the control group was $(59.72 \pm 12.37)$, with no statistically significant difference between the two groups $(t=1.69, p>.05)$. There were 42 males and 46 females in the experimental group, and 48 males and 40 females in the control group. There was no statistically significant difference in gender composition between the two groups $\left(\chi^{2}=0.82, p>.05\right)$.

Before treatment, the levels of fasting blood glucose, twohour postprandial blood glucose, glycosylated hemoglobin and depression index in the experimental group were not significantly different from those in the control group ( $p$ $>$.05); after 3-month treatment, the levels of FPG, PG2h, HbA1c and depression index were significantly lower than those in the control group, the difference was statistically significant $(p<.01)$; in 2 months after intervention stopped, the levels of FPG, PG2h, HbA1c and depression index in the experiment group were still lower than those in the control group, the difference was statistically significant $(p<.01$, see Table 1).

Table 1: Comparison of glucose metabolism indicators and depression index in 2 groups of patients with diabetes at each time point $(\bar{x} \pm s)$

\begin{tabular}{|c|c|c|c|c|c|c|c|}
\hline \multirow[b]{2}{*}{ Group } & \multirow[b]{2}{*}{$\mathbf{n}$} & \multicolumn{3}{|c|}{ FPG (mmol/L) } & \multicolumn{3}{|c|}{ PG2h (mmol/L) } \\
\hline & & $\begin{array}{l}\text { Before } \\
\text { Treatment }\end{array}$ & $\begin{array}{l}\text { After 3-month } \\
\text { treatment }\end{array}$ & $\begin{array}{l}\text { In } 2 \text { months after } \\
\text { intervention stopped }\end{array}$ & $\begin{array}{l}\text { Before } \\
\text { Treatment }\end{array}$ & $\begin{array}{l}\text { After 3-month } \\
\text { treatment }\end{array}$ & $\begin{array}{l}\text { In } 2 \text { months after } \\
\text { intervention stopped }\end{array}$ \\
\hline $\begin{array}{l}\text { Experimental } \\
\text { Group }\end{array}$ & 88 & $10.01 \pm 1.26$ & $6.70 \pm 0.71$ & $6.90 \pm 0.58$ & $15.95 \pm 1.10$ & $9.50 \pm 1.02$ & $10.51 \pm 0.85$ \\
\hline Control Group & 88 & $9.80 \pm 1.20$ & $7.39 \pm 0.74$ & $7.38 \pm 0.65$ & $15.82 \pm 1.08$ & $12.65 \pm 1.41$ & $12.17 \pm 1.11$ \\
\hline$t$ value & & 1.13 & 6.36 & 5.16 & 0.77 & 17.00 & 11.12 \\
\hline$p$ value & & .26 & $<.01$ & $<.01$ & .44 & $<.01$ & $<.01$ \\
\hline \multirow[b]{2}{*}{ Group } & \multirow[b]{2}{*}{$\mathbf{n}$} & \multicolumn{3}{|c|}{ HbA1c (\%) } & \multicolumn{3}{|c|}{ Depression Index } \\
\hline & & $\begin{array}{l}\text { Before } \\
\text { Treatment }\end{array}$ & $\begin{array}{l}\text { After 3-month } \\
\text { treatment }\end{array}$ & $\begin{array}{l}\text { In } 2 \text { months after } \\
\text { intervention stopped }\end{array}$ & $\begin{array}{l}\text { Before } \\
\text { Treatment }\end{array}$ & $\begin{array}{l}\text { After 3-month } \\
\text { treatment }\end{array}$ & $\begin{array}{l}\text { In } 2 \text { months after } \\
\text { intervention stopped }\end{array}$ \\
\hline $\begin{array}{l}\text { Experimental } \\
\text { Group }\end{array}$ & 88 & $8.10 \pm 0.52$ & $5.85 \pm 0.13$ & $6.13 \pm 0.28$ & $0.60 \pm 0.02$ & $0.52 \pm 0.01$ & $0.54 \pm 0.02$ \\
\hline$t$ value & & 1.75 & 34.44 & 18.98 & 0.67 & 22.00 & 10.00 \\
\hline$p$ value & & .08 & $<.01$ & $<.01$ & .40 & $<.01$ & $<.01$ \\
\hline
\end{tabular}

\section{Discussion}

T2DM patients account for more than $90 \%$ of patients with diabetes mellitus. This disease not only manifests metabolic disorders characterized by increased level of blood glucose, but also easily leads to depression, anxiety and other psychological disorders. ${ }^{[3]}$ Depression symptoms arise from physical diseases and affect all stages of them. Studies have shown that ${ }^{[4]}$ psychological problems can arise from cardiovascular disease, and clinical manifestations are depression, anxiety and so on. Compared to patients with non-cardiovascular diseases, the incidence of depression in patients with cardiovascular diseases is increased to $10 \%$ $40 \%$, and the incidence of anxiety is increased to $20 \%-70 \%$. It has been reported that ${ }^{[5]}$ the incidence of depression in diabetic patients is higher than that in the normal popula- tion. Jin $\mathrm{Y}$ et al. ${ }^{[6]}$ have pointed out that the incidence of depression in T2DM patients is $34.8 \%-52.6 \%$ and this is 3.0-4.6 times higher than that in the general population. In this study, T2DM patients selected from the community were taken as the research objects, remarkably representative in comparison with diabetic patients in the conventional hospitals; after independently rating by the depression scale, it was found that the incidence of depression in T2DM patients was $21.4 \%$, slightly lower than that reported by Zhao $\mathrm{J}$ et al. $(31.9 \%)^{[7]}$ and Zhang $\mathrm{R}$ et al. (34.1\%). ${ }^{[8]}$ In recent years, meta-analysis results in the longitudinal or prospective studies conducted by foreign scholars such as Park M et al. ${ }^{[9]}$ have shown that the morality risk in T2DM patients with depression is 1.5 times higher than that in the non-depression population. In the traditional diagnosis and treatment process of type 2 diabetes, it is common to only 
emphasize the control of blood glucose rather than the quality of life. The guidelines established by American Diabetes Association (2012) have emphasized that psychological health is an essential part of diabetes management and should be assessed throughout the process of diabetic treatment. The main reasons are as follows: first, diabetes is a chronic metabolic disease that requires long-term changes in lifestyle, meanwhile, anxious or depressive symptoms consequently arise from the loss in life, family, career and many other aspects; secondly, the risk of diabetes is increased after activating the axis of hypothalamus-hypophysis-adrenal glands and sympathetic nervous system. Studies have found that the percentage of senile T2DM patients with depression is higher than national norm $(33.46 \pm 8.55),{ }^{[10,11]}$ the cause may be that senile T2DM patients suffer from gradual degeneration of tissue function and have to reduce the time and the amount of work (or other activities) with adaptive capacity decreased due to various factors. Patients negatively assess their overall health conditions, considering them to be more likely to get sick or worse than before; negative emotions often arise eventually, such as black mood, exhaustion or unhappiness. Besides, depressive symptoms easily arise in senile T2DM patients due to negative life events, such as changes in long-term persistent lifestyle as well as drug therapy, medical expenses, income decrease etc. Studies ${ }^{[12]}$ have shown that, it should be noted that T2DM patients need to be given psychological counseling, especially those who are aged, with long-term course of disease, well-educated as well as with high FPG, ALU and multiple complications. Meanwhile, it is recommended to make a diet intervention as early as possible. Psychotherapy is defined as a process that clinicians can establish a good doctor-patient relationship with patients, with the application of relevant psychological and medical knowledge by verbalized or unverbalized communication, so that patients are willing to overcome and correct negative lifestyles. Some researches also have shown that, ${ }^{[13]}$ health education, psychological nursing and traditional drug therapy are identically important in the diabetic treatment. Humanized health education measures can effectively improve the negative emotions arising in T2DM patients.

The researchers in this study consist of community general doctors, nurses, pharmacists, physicians and psychologists. All participants are proficient in the research pro-

\section{References}

[1] Wang Y. Practice of Geriatrics. Beijing: People's Medical Publishing House; 2013. 151 p.

[2] Chinese Diabetes Society of Chinese Medical Association. China Guideline for Type 2 Diabetes (2013 Edition). Beijing: Peking University Medical Press; 2014. 67 p.

[3] Jin MJ, Chen GT, Huang J, et al. Effect of health education on T2DM patients in psychological state and treatment compliance. cess. The form of psychotherapy used in the task design is called group therapy, ${ }^{[14]}$ i.e., there is no kinship between patients with same diseases or disorders and identical treatment is required. It is recommended to establish anti-diabetes club, hold lectures and video sessions, and distribute diabetes-related handbooks. Diabetes physicians instruct diabetes-related knowledge to diabetic patients first, and then communicate with patients specifically based on the results of psychological tests. Patients communicate with each other, help each other, and inspire those who run into negative emotions deeply. Doctors timely and effectively answer patients' questions, so that patients fully feel concerns and warmth. On the basis of integrated assessments performed to patients, comprehensive intervention measures were taken individually and specifically to alleviate or eliminate the negative emotions arising in patients, improve the treatment compliance in patients and their families, and promote the recovery of patients' functions. Compared with hospitals in the traditional sense, the community team has multiple functions such as medical care, prevention, health care, rehabilitation and health education, and can comprehensively carry out scientific assessments to patients with diabetes from all sides. To deal with diabetic patients with a high risk of depression or diabetic patients with depressive symptoms already, it should start from the psycho-social model to alleviate or eliminate adverse factors, adjust the treatment plan in a human-friendly manner, actively control blood glucose, prevent complications and further improve the quality of life and prolong life span. It is expected to continue the hospital-community-family disease management model ${ }^{[15]}$ and make full use of medical resources, to set up a completely social knowledge framework, relieve the burdens on families, establish an effective social support system and improve patients' life quality. If depressive symptoms are more serious, it is recommended to make referrals to specialized hospitals for further control of the disease. This study has showed that, glucose metabolism indicators and depressive symptoms can be effectively controlled through active psychological intervention, which is of good economic and social benefits and worthy of being promoted.

\section{Conflicts of Interest Disclosure}

The authors have no conflicts of interest related to this article.

Zhejiang Medical Journal. 2012; 37(7): 570-571.

[4] $\mathrm{Hu} \mathrm{CY}$. Application of psycho-cardiology medical mode in patients of unstable angina complicated with depression and anxiety. Anhui Medical Journal. 2014; 35(12): 1648-1650.

[5] Liu K, Ning XY, Zhou Q, et al. Analysis on related factors of T2DM patients with depression in Shunde region of Guangdong. Contemporary Medicine. 2013; 12(5): 3-5.

[6] Jin Y, Sun XR, Huang Y, et al. Relationship between BDNF and depression with type 2 diabetes mellitus. Nervous Diseases and Mental 
Health. 2015; 31(2): 158-160.

[7] Zhao J, Lou PA, Zhang P, et al. The risk factors for anxiety and depression in patients with type 2 diabetes in Xuzhou. Chinese Journal of Diabetes. 2014; 7(22): 615-616.

[8] Zhang R, Dai W, Ye J, et al. Effect of psychotherapy on patients with type 2 diabetes and light-moderate degree depression. Anhui Medical Journal. 2013; 34(9): 1285-1287.

[9] Park M, Katon WJ, Wolf FM. Depression and risk of mortality in individuals with diabetes: a meta-analysis and systematic review. Gen Hosp Psychiatry. 2013; 35(3): 217-225. PMid: 23415577. https://doi.org/10.1016/j.genhosppsych.2013.01.006

[10] Lin T, Liu XL, Lan YT, et al. Depression degree and related factors in senile T2DM patients. Chinese Journal of Gerontology. 2013;
33(9): 2115-2117.

[11] Wang XD, Wang XL, Ma H. Psychological Health Rating Scale Handbook. Beijing: Chinese Mental Health Journal; 1999. 2356 p.

[12] Fan HG. Analysis on depression degree and related factors in T2DM patients. Modern Practical Medicine. 2015; 27(5): 606-607.

[13] Wang F. Effects of health education and psychological intervention in the long-term treatment of diabetes. Jilin Medical Journal. 2014; 35(25): 5730-5731.

[14] Chen HZ, Lin GW. Practice of Internal Medicine. $13^{\text {th }}$ Edition. Beijing: People's Medical Publishing House; 2009. 2992-2993 p.

[15] Gong J, Zheng HY, Wang LL, et al. Research progress on controllable influencing factors of type 2 diabetes with mild cognitive disorders. Anhui Medical Journal. 2016; 37(2): 237-239. 